\title{
Correction: Alteration of urinary neutrophil gelatinase-associated lipocalin as a predictor of tacrolimus-induced chronic renal allograft fibrosis in tacrolimus dose adjustments following kidney transplantation
}

\author{
The PLOS ONEStaff
}

The first two authors, Wiwat Chancharoenthana and Asada Leelahavanichkul, should be noted as equal co-corresponding authors.

There is an error in the Funding statement. The correct Funding statement is as follows: All of the funding supports during study period including the Development of New Faculty Staff fund and Ratchadapiseksomphot Endowment Fund 2017 (76001-HR), Faculty of Medicine, Chulalongkorn University and National Science and Technology Development Agency (NSTDA: P-13-00505)-Dr. Asada Leelahavanichkul. A.L. is under Center of Excellence in Immunology and Immune Mediated Diseases group. The funders had no role in study design, data collection and analysis, decision to publish, or preparation of the manuscript.

The publisher apologizes for the errors.

\section{Reference}

1. Chancharoenthana $W$, Leelahavanichkul $A$, Wattanatorn $S$, Avihingsanon $Y$, Praditpornsilpa K, EiamOng S, et al. (2018) Alteration of urinary neutrophil gelatinase-associated lipocalin as a predictor of tacrolimus-induced chronic renal allograft fibrosis in tacrolimus dose adjustments following kidney transplantation. PLoS ONE 13(12): e0209708. https://doi.org/10.1371/journal.pone.0209708 PMID: 30576367

\section{f OPEN ACCESS}

Citation: The PLOS ONEStaff (2019) Correction: Alteration of urinary neutrophil gelatinaseassociated lipocalin as a predictor of tacrolimusinduced chronic renal allograft fibrosis in tacrolimus dose adjustments following kidney transplantation. PLOS ONE 14(2): e0213009. https://doi.org/10.1371/journal.pone.0213009

Published: February 22, 2019

Copyright: @ 2019 The PLOS ONE Staff. This is an open access article distributed under the terms of the Creative Commons Attribution License, which permits unrestricted use, distribution, and reproduction in any medium, provided the original author and source are credited. 